\title{
Multi-dimension and Comprehensive Assessment on the Utilizing and Sharing of Regional Large-scale Scientific Equipment
}

\author{
Chen $\mathrm{Li}^{*}, \mathrm{Lv}$ Yongbo and Chen Chi
}

School of Traffic and Transportation, Beijing Jiaotong University, Beijing 100044, China

\begin{abstract}
Based on the data from 30 provincial regions in China, an assessment and empirical analysis was carried out on the utilizing and sharing of the large-scale scientific equipment with a comprehensive assessment model established based on three dimensions, namely, equipment, utilization and sharing. The assessment results were interpreted in light of relevant policies. The results showed that on the whole, the overall development level in the provincial regions in eastern and central China is higher than that in western China. This is mostly because of the large gap among the different provincial regions with respect to the equipped level. But in terms of utilizing and sharing, some of the Western provincial regions, such as Ningxia, perform well, which is worthy of our attention. Policy adjustment targeting at the differentiation, elevation of the capacity of the equipment management personnel, perfection of the sharing and cooperation platform, and the promotion of the establishment of open sharing funds, are all important measures to promote the utilization and sharing of the large -scale scientific equipment and to narrow the gap among different regions.
\end{abstract}

Keywords: Large-scale scientific equipment, utilization, sharing, 3D assessment model.

\section{INTRODUCTION}

Along with the coming of the age of knowledge of economy and globalization, scientific resources that support the innovation of the whole society are becoming important national strategic resources. The Outline of the National Long-term Scientific and Technological Development Plan (2006-2020) points out that it is the optimization and restructure of the fundamental scientific conditions that are the important national infrastructures. This should also be a new focus of the international competition in scientifictechnological innovation [1]. After long-term efforts, China's scientific and technological infrastructures have greatly improved. A large number of advanced scientific equipment have been introduced from abroad. Large-scale scientific equipment is featured by advanced technology, rapid upgrading, high prices and high-tech. If the life cycle of a piece of equipment is divided into three phases, namely advanced, comparable and lag-behind, generally, only less than one-third is in the advanced phase [2]. Thus, only sufficient utilization and sharing could guarantee the utilization of the advanced phase and maximize the value of the large-scale scientific equipment. Otherwise, the equipment will be wasted. If that happens, China's level of scientific research would be greatly restricted. However, the utilization of large-scale scientific equipment in China is far from satisfying the demand of scientific research. There are

*Address correspondence to this author at the School of Traffic and Transportation, Beijing Jiaotong University, Beijing 100044, China; E-mails: aaa19850721@163.com many problems remaining to be solved, among which low utilization, poor allocation, scattered distribution and unreasonable sharing mechanism are the most pronounced.

Although achievements have been made in the construction and utilization of the large-scale scientific equipment in China in the past decades, the problems such as scattered allocation and low efficiency operation still exist. Other problems include lack of guidance from law and regulations, lack of a sharing platform, lack of a sharing awareness, obstructed channels for the sharing of scientific resources, and lack of talent cultivation mechanism. The status of utilization of large-scale scientific equipment in China has been analyzed in previous studies. Liu (2001) discussed the rational allocation and sharing of the equipment resources based on the conditions of limited investments in the provincial universities and colleges [3]. Xia et al. (2005) [4] established a well-functioning equipment resources mechanism throughout the province with sharing as the core together with the "Three Operating Mechanisms" and "Five Safeguards". Xu et al. (2010) [5] put forward a spatial analysis method for the utilization status analysis of the large-scale scientific equipment. $\mathrm{Hu}$ et al. analyzed the widespread problems related to the large-scale scientific equipment management in colleges and universities. They pointed out the need for building reasonable indices for the utilizing and sharing of the equipment. Zhang et al. (2013) [6] established a rank-sum model, and quantitatively assessed the utilization of largescale equipment in China. 
Moreover, when calculating the equipment utilization rate and external service rate, there is usually no weighted calculation of original value of equipment. However, the result is that the equipment with equal length of working hours may end up with the same contribution rate. As we all know, the performance of "elephant" and "ants" is dramatically the difference within the same period of time. However, the current evaluation of utilization and sharing of large-scale scientific equipment does not consider the original values of the equipment.

Therefore, it is necessary to comprehensively evaluate the utilization and sharing of China's large-scale scientific equipment from multiple dimensions. This is important to providing policy suggestions for the future development of large-scale scientific equipment. It is also the focus of the present study.

\section{ANALYSIS OF CHINA'S CURRENT DEVELOPMENT SITUATION OF LARGE- SCALE SCIENTIFIC EQUIPMENT}

In the five years from 2008 to 2012 , the number of largescale scientific equipment in China had rose from 21496 to 43028. The total original value of the equipment increased from 28.799 billion yuan to 57.167 billion yuan (left in Fig. 1). Both the number and original value of the equipment had more than doubled. The average original values of the equipment were about 1.3 million RMB, without an obvious increase. The annual average working hours of the equipment fluctuated around $1200 \mathrm{~h} / \mathrm{set}$ (middle in Fig. 1). There was neither an increase nor a decrease. The annual average external service hours, as an index of the sharing status of equipment, underwent a dramatic decline instead of an increase (right in Fig. 1). It decreased from $236.6 \mathrm{~h} / \mathrm{set}$ in 2008 to $167.13 \mathrm{~h} /$ set in 2012 , by as high as $29.4 \%$.

The number of large-scale scientific equipment is higher in eastern China than in western China. As shown by the original values of equipment in different provinces in 2012, the top five provinces or municipalities directly under Central Government were Beijing, Shanghai, Guangdong, Jiangsu, Shandong and Zhejiang, all in eastern China. The last five provinces were Jiangxi, Hainan, Ningxia, Qinghai and Inner Mongolia, in which the last three belong to western China. The distribution of large-scale scientific equipment across the provinces is extremely non-uniform. Beijing ranks the first, with the original value of equipment exceeding 10 billion, which was more than doubled the value in Shanghai in the second place. Beijing's original value of equipment was 285 times that of Inner Mongolia ranking the last. One of the important reasons lies in the lack of reasonable method to evaluate the local development status of large-scale scientific equipment. This results in an unbalanced equipment allocation and pronounced Matthew effect. Therefore, we establish a set of evaluation indices and multi-dimensional evaluation model to comprehensively analyze the sharing status and to provide reference for technology management decision-making.

\section{CONSTRUCTION OF SYSTEM OF INDICES FOR UTILIZATION AND SHARING OF LARGE-SCALE SCIENTIFIC EQUIPMENT AND GRADING}

\subsection{Definition of Research Scope}

Large-scale scientific equipment mentioned here refers to the scientific equipment with original value higher than 500 thousand RMB [7]. Platform Center of the Ministry of Science and Technology carried out survey, classification and analysis of China's utilization and sharing of large-scale scientific equipment in the past a few consecutive years. The data were screened. The large-scale engineering equipment at the affiliated base, those with annual average working hours exceeding $8640(360 * 24)$, computers and auxiliaries, and equipment to be scraped, were excluded [8]. By the end of 2012, China had 43028 sets of large-scale scientific equipment in total, with the original value of as high as 57.17 billion RMB. They are distributed at 524 legal entities such as Chinese Academy of Sciences and Peking University as well as 39 national-level administrative departments such as Ministry of Education and Ministry of Industry and Information.

Here "utilization rate" of scientific equipment refers to the ratio of working hours of equipment to rated working

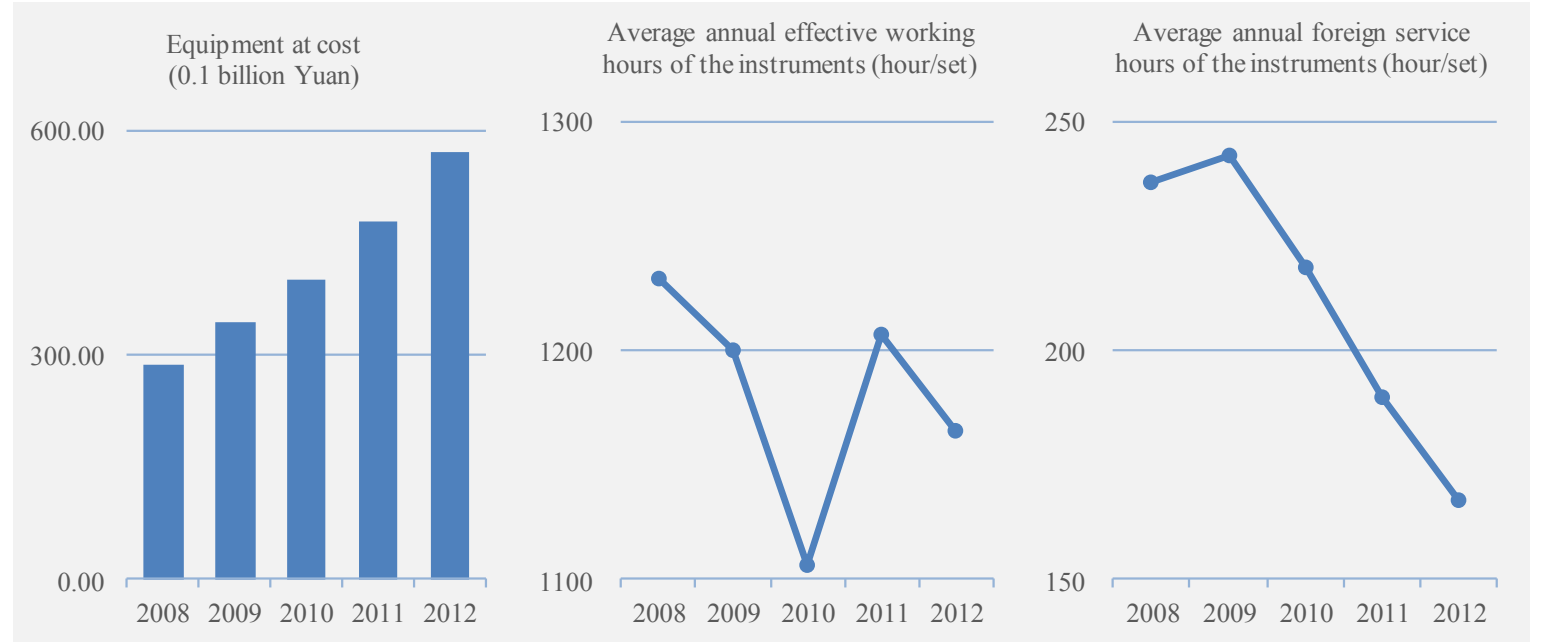

Fig. (1). Utilization and sharing of large-scale scientific equipment in China (2008-2012). 
hours within a specific time period. The annual rate working hours of the equipment is set as $8 \mathrm{~h} * 200 \mathrm{~d}=1600 \mathrm{~h}$. In that case, the utilization rate of scientific equipment will exceed $100 \%$. Therefore, we treat the calculated utilization rate above $100 \%$ as $100 \%$. But this method will artificially reduce the differences between the utilization efficiencies of scientific equipment across the regions. So we do not make special treatment on utilization rate larger than $100 \%$.

Furthermore, for the convenience of data acquisition, we only process data of large-scale scientific equipment in 30 provinces excluding Tibet, Hong Kong, Macau and Taiwan.

\subsection{Construction of System of Indices}

The indices of utilization and sharing of large-scale scientific equipment can reflect the status of scientific equipment utilization and sharing comprehensively. For each dimension, 3 secondary indices are selected to constitute an index system. The weight of each index is determined by expert grading.

(1) Equipped capacity of large-scale scientific equipment

Equipped level measures the scale, structure, development, allocation, R \& D and centralized management of large-scale scientific equipment in a specific region.

Original value $a_{11}$ of equipment (ten thousand RMB): it is the sum of original value of all equipment in a specific region, and it reflects the equipment construction scale of this region. It also represents the potential of this region in equipment utilization and sharing, with the weight of 0.3 .

Original value $a_{12}$ of newly added equipment (ten thousand RMB): it is the sum of original value of all newly added large-scale scientific equipment in a specific region in the past 3 years. The index reflects the equipment input scale of this region in the past 3 years as well as the equipment construction \& input capacity, with the weight of 0.3 .

Original value $a_{13}$ per staff engaged in scientific research (ten thousand $\mathrm{RMB}$ /per person): it is the original value of equipment in a specific region divided by the number of staff engaged in scientific research (here it does not refer to all people engaged in scientific research of the region). This index measures the degree of allocation of equipment among users in this region, with the weight of 0.4 .

\section{(2) Ability of utilization of large-scale scientific equipment}

Utilization level comprehensive measures the status of utilization of large-scale scientific equipment in a specific region (including the utilization efficiencies of all equipment, newly added equipment and high-end equipment, as well as the total working hours, full-load operations and normal operations.

Utilization rate $a_{21}$ of equipment (\%): the utilization rate of a single equipment is the ratio of annual working hours to annual rated working hours of an equipment (the annual rated working hours is $1600 \mathrm{~h}$, that is, 8 hours for 200 days every year). The utilization efficiency of equipment in a specific region is the weighted sum of utilization rate of a single equipment according to the proportion of original value of the equipment. This index reflects the overall utilization efficiency of equipment in this region. It has the weight of 0.3 .

Utilization rate $a_{22}$ of newly added equipment (\%): the utilization rate of newly added equipment in a specific region in the past 3 years is the weighted sum by the proportion of original value. The index reflects the utilization efficiency of newly added equipment in this region, with the weight of 0.3 .

Proportion $a_{23}$ of equipment operating under full load $(\%)$ : it is the proportion of equipment with annual working hours equal or exceeding the rated working hours. This index reflects the situation of high-level operation of equipment in this region, with the weight of 0.4 .

\section{(3) Capacity of sharing large-scale scientific equipment}

Sharing level comprehensively measures the situation of external service rate (including the external service rates of total equipment and newly added equipment), total hours of external services, proportion of equipment with information publicity, average income from equipment services and regional sharing. It has the weight of 0.3 .

External service rate $a_{31}$ of equipment (\%): the external service rate of a single equipment is the proportion of external service hours of a single equipment in annual rated service working hours. The external service rate of a specific region is the weighted sum of external service rate of a single equipment by the proportion of its original value. This index reflects the overall external service level of the region, with the weight of 0.3 .

External service rate $a_{32}$ of newly added equipment (\%): the external service rate of newly added equipment in the past 3 years is the weighted sum by the proportion of its original value. This index measures the external service level of the newly added equipment in this region.

Proportion $a_{33}$ of equipment shared regionally (\%): it is the proportion of original value of equipment for crossregional external services [9]. The index measures the status of cross-regional sharing of the equipment in this region, with the weight of 0.4 .

\subsection{Non-Dimensionalization}

Evaluation based on multiple indices involves the actual values and assessment values of each index. The two values are different in dimension. Non-dimensionalization is to remove the differences in measurement units and the differences in order of magnitude. After nondimensionalization, the indices can be synthesized. Benchmarking method is employed for nondimensionalization of secondary indices, and the secondary index $x_{i j}$ is obtained.

$$
x_{i j}^{k}=\frac{a_{i j}^{k}}{\max _{k}\left\{a_{i j}^{k}\right\}} \times 100 \quad i=1,2,3 \quad j=1,2,3 \quad k=1,2, \ldots, 30
$$

Then, integrated weighing is employed to calculate the primary index $y_{i}^{k}$.

$$
y_{i}^{k}=\sum_{j=1}^{3} x_{i j}^{k} \times \gamma_{i j} \quad i=1,2,3 \quad k=1,2, \ldots, 30
$$


where $\gamma_{i j}$ is the weight of each secondary index, which is determined by expert grading.

\section{THREE-DIMENSIONAL EVALUATION MODEL (EQUIPMENT-UTILIZATION-SHARING)}

\subsection{Model Construction}

The influence of the above-mentioned factors on the sharing of large-scale scientific equipment is evaluated and compared. Clustering is adopted for hierarchical assessment of the influence factors of three aspects.

The elements to be assessed are divided into $p$ categories. Two most distant elements $x_{i_{1}}$ and $x_{i_{2}}$ are selected as the first two clustering points. That is to say, to choose $x_{i}$ and $x_{i_{2}}$ that makes $d\left(x_{i_{1}}, x_{i_{2}}\right)=d_{i_{1} i_{2}}=\max \left\{d_{i j}\right\}$.

Then the third clustering point is selected that makes the shortest distance from $x$ to the first clustering points equal to the largest one among the shorter distance from $x_{i_{1}}$ and $x_{i_{2}}$ :

$\min \left\{d\left(x_{i_{3}}, x_{i_{r}}\right) \quad r=1,2\right\}=\max \left\{\min \left[d\left(x_{j}, x_{i_{r}}\right) \quad r=1,2\right], j \neq i_{1}, i_{2}\right\}$

By the same principle, $x_{i_{4}}$ is selected, until $k$ clustering points are selected, namely, $x_{i_{1}}, x_{i_{2}}, \ldots, x_{i_{4}}$.

The selection process can be completed using recursion formula. If $l$ clustering points are selected, then the principle for choosing the $l+1$-th clustering point can be expressed as

$\min \left\{d\left(x_{i_{i_{1}}}, x_{i_{r}}\right) \quad r=1,2, \mathrm{~L}, l\right\}=\max \left\{\min \left[d\left(x_{j}, x_{i_{r}}\right) \quad r=1,2, \mathrm{~L}, l\right], j \neq i_{1}, \mathrm{~L}, i_{r}\right\}$

In the above clustering process, we select 3 clustering points for each category of influence factors, i.e. $p=3$. Therefore, the equipped level is divided into high, moderate and low. The same applies to the grading of utilization level and sharing level.

Based on the analysis of equipped level, utilization level and sharing level of large-scale scientific equipment, the
Hall's three-dimensional structure in system engineering methodology is referred to. The three-dimensional model for evaluating the utilization and sharing of the equipment is constructed as shown in the Fig. (2).

In the above figure, the factors of three dimensions, namely, supply, operation and sharing construction of largescale scientific equipment, are assigned with different scores. Thus, the utilization and sharing status of equipment are plotted under the coordinate system. Points A, B and C in the figure stand for this.

A: $\left(X_{1}, Y_{1}, Z_{1}\right)=$ (weak equipped capacity, weak operating status, weak sharing construction capacity $)=(1,1,1)$

B: $\left(X_{2}, Y_{2}, Z_{2}\right)=$ (moderate equipped capacity, moderate operating status, moderate sharing construction $)=(2,2,2)$

C: $\left(X_{3}, Y_{3}, Z_{3}\right)=$ (strong equipped capacity, good operating status, good sharing construction $)=(3,3,3)$

If the three coordinates are synthesized by multiplication, then the synthetic scores of equipment utilization and sharing in a specific region are obtained and expressed as $\delta$. Then,

$\delta_{(i, j, k)}=f($ Equipment Level $(i)$, Utilization Level $(j)$,Sharing Level $(k))=Z_{i}{ }^{*} L_{j}{ }^{*} G_{k}(*)$

In the formula of synthetic score proposed in this article, different elements represent different types, where $Z$ represents the dimension of equipped level; $Z_{1}, Z_{2} 、 Z_{3}$ represent weak, moderate and strong levels, respectively. $L$ represents the dimension of utilization; $L_{1} 、 L_{2} 、 L_{3}$ represent poor, moderate and good sharing construction capacity; $G$ represents the dimension of sharing; $G_{1}, G_{2} 、 G_{3}$ represent poor, moderate and good sharing construction capacity, respectively. Thus, theoretically, $\delta_{(i, j, k)}$ has 27 combinations, with 10 different values.

By $\delta_{(i, j, k)}$, the equipment utilization and sharing status across the provinces is divided into different intervals; the

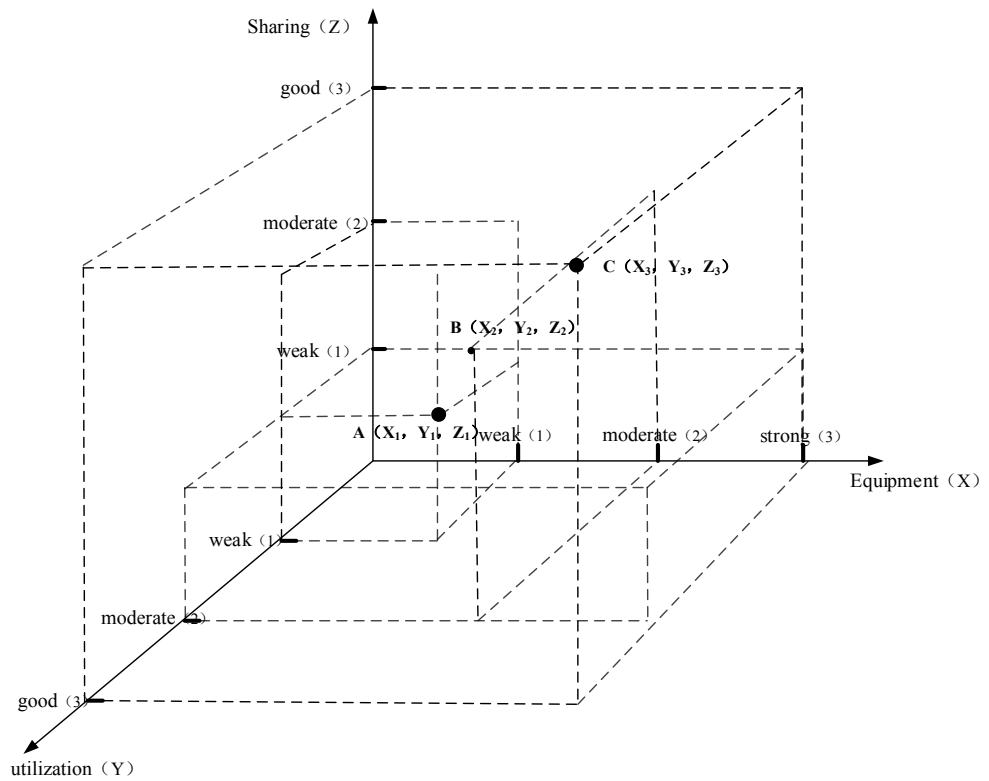

Fig. (2). Three-dimensional model for the evaluation of utilization and sharing status of large-scale scientific equipment. 
provinces with $1 \leq \delta \leq 3$ are classified as category I; the provinces with $4 \leq \delta \leq 9$ are classified as category II; the provinces with $10 \leq \delta \leq 27$ are classified as category III.

\subsection{Empirical Test Results}

According to the aforesaid formulas and threedimensional evaluation model of the utilizing and sharing status, the development level of equipment, utilization and sharing in 30 provincial areas throughout China in 2012 was assessed. Please refer to Table 1(vide infra) for results.
Based on the grading results of all provincial areas in such three dimensions as equipment, utilization and sharing in Table 1, we classified the results by $\delta$ value according to (*) formula. The result is presented in Table 2.

On the basis of calculation and classification results in Figs. (1 and 2), we can reach the following conclusions:

(1) As shown by the classification by value, the number of provincial regions that are included into Category $\mathrm{I}$ is 6 . They can be further divided into two sub-categories. Beijing and Shanghai, the two municipalities directly under the Central Government, are the first category as

Table 1. Indices of utilization and sharing of large-scale scientific equipment in China's Provincial Areas in 2012.

\begin{tabular}{|c|c|c|c|c|c|c|c|}
\hline Serial Number & Region & Equipment Level Z & Grading & Utilization Level L & Grading & Sharing Level G & Grading \\
\hline 1 & Beijing & 100.0 & 1 & 62.2 & 1 & 22.3 & 2 \\
\hline 2 & Tianjin & 26.0 & 2 & 62.4 & 1 & 16.8 & 3 \\
\hline 3 & Hebei & 11.8 & 3 & 44.1 & 2 & 6.9 & 3 \\
\hline 4 & Shanxi & 8.3 & 3 & 73.1 & 1 & 9.1 & 3 \\
\hline 5 & Inner Mongolia & 11.9 & 3 & 29.7 & 3 & 1.6 & 3 \\
\hline 6 & Liaoning & 29.0 & 2 & 63.4 & 1 & 19.9 & 2 \\
\hline 7 & Jilin & 24.5 & 2 & 77.9 & 1 & 19.2 & 2 \\
\hline 8 & Heilongjiang & 15.5 & 3 & 31.6 & 3 & 13.3 & 3 \\
\hline 9 & Shanghai & 66.3 & 1 & 70.4 & 1 & 35.9 & 2 \\
\hline 10 & Jiangsu & 31.0 & 2 & 55.5 & 2 & 21.9 & 2 \\
\hline 11 & Zhejiang & 30.0 & 2 & 48.4 & 2 & 37.3 & 2 \\
\hline 12 & Anhui & 14.2 & 3 & 86.9 & 1 & 27.0 & 2 \\
\hline 13 & Fujian & 15.0 & 3 & 38.6 & 2 & 14.4 & 3 \\
\hline 14 & Jiangxi & 7.1 & 3 & 41.4 & 2 & 13.0 & 3 \\
\hline 15 & Shandong & 22.0 & 2 & 67.9 & 1 & 33.2 & 2 \\
\hline 16 & Henan & 14.0 & 3 & 40.6 & 2 & 19.3 & 2 \\
\hline 17 & Hubei & 25.2 & 2 & 45.1 & 2 & 27.1 & 2 \\
\hline 18 & Hunan & 11.1 & 3 & 60.5 & 1 & 49.7 & 1 \\
\hline 19 & Guangdong & 53.9 & 1 & 53.9 & 2 & 31.3 & 2 \\
\hline 20 & Guangxi & 11.7 & 3 & 46.9 & 2 & 35.4 & 2 \\
\hline 21 & Hainan & 14.2 & 3 & 21.2 & 3 & 3.7 & 3 \\
\hline 22 & Chongqing & 15.4 & 3 & 54.8 & 2 & 24.5 & 2 \\
\hline 23 & Sichuan & 14.5 & 3 & 64.8 & 1 & 19.0 & 2 \\
\hline 24 & Guizhou & 6.8 & 3 & 50.1 & 2 & 50.3 & 1 \\
\hline 25 & Yunnan & 12.0 & 3 & 79.2 & 1 & 57.2 & 1 \\
\hline 26 & Shaanxi & 19.5 & 2 & 49.8 & 2 & 16.2 & 3 \\
\hline 27 & Gansu & 19.9 & 2 & 57.2 & 2 & 28.7 & 2 \\
\hline 28 & Qinghai & 6.0 & 3 & 67.9 & 1 & 6.4 & 3 \\
\hline 29 & Ningxia & 6.9 & 3 & 75.7 & 1 & 66.5 & 1 \\
\hline 30 & Xinjiang & 7.9 & 3 & 63.6 & 1 & 56.7 & 1 \\
\hline
\end{tabular}


Table 2. Evaluation interval of sharing status of large-scale scientific instruments.

\begin{tabular}{|c|c|l|}
\hline Interval & Category & \multicolumn{1}{|c|}{ Region } \\
\hline \hline $1 \leq \delta \leq 3$ & Category I & Beijing, Shanghai, Hunan, Yunnan, Ningxia, Xinjiang \\
\hline $4 \leq \delta \leq 9$ & Category II & Henan, Guangxi, Chongqing, Gansu, Hebei, Fujian, Jiangxi, Shaanxi, Inner Mongolia, Heilongjiang, Hainan \\
\hline $12 \leq \delta \leq 27$ & Category III & Henan, Guangxi, Chongqing, Gansu, Hebei, Fujian, Jiangxi, Shaanxi, Inner Mongolia, Heilongjiang, Hainan \\
\hline
\end{tabular}

they enjoy considerable advantages over other provincial areas in economy, science and technology, culture and other fields. In terms of large-scale scientific equipment, the two cities enjoy a more balanced development in all aspects. Especially, Beijing, with so many universities and research institutes, equips itself with abundant advanced large-scale scientific instruments that other provincial areas cannot catch up with. Another subcategory consists of Hunan, Yunnan, Ningxia and Xinjiang, in central and western China. It is not difficult to find by carefully analyzing their index values in the three dimensions that the major reason why they can be included in Category I is their high level of utilization and sharing, suggesting that developed economy and huge amount of large-scale scientific equipment do not mean high level of utilization and sharing. A good equipment sharing platform as well as high quality research and managerial staff members are the key aspects of the utilization and sharing of equipment.

(2) By analyzing the regions that are classified into Categories II and III, we can discover that the development of large-scale scientific equipment in central China is better than that in western regions. The equipped level of largescale scientific equipment in different provincial areas differs greatly, but the utilization and sharing levels are relatively balanced. We can see that the equipped level index in provincial areas that are classified into Category III is not higher than 20, which is also the major difference existing between them and Category III regions. From this we can conclude that the raising of absolute strength is urgently needed by the western areas where economy, science and technology are underdeveloped. Therefore, science and technology management organs should take them into consideration when allocating resources and making policies.

(3) The development status of large-scale scientific equipment in some particular or typical regions among the 30 provincial areas has its own characteristics, which are worthy of consideration. One of the typical regions is Beijing. The equipped level of large-scale scientific equipment in Beijing is the highest but the utilization index only ranks the 13 th among the 30 , and the sharing index, the No. 15, both belonging to a moderate level. Although the total quantity of equipment is huge, there is redundancy of equipment and a low request for sharing; secondly, due to the lack of effective equipment sharing platform, there is a weak connection between owners and demanding parties. A considerable amount of equipment is left idle and wasted. Thirdly, the management professionals who have varying levels of skills and knowledge cannot provide proper maintenance for the equipment or make full use of the equipment.

(4) Another typical region is Ningxia. In 2012, the original value of large-scale scientific equipment of Ningxia was only $91,730,000$ yuan, less than $1 \%$ of that of Beijing. However, since 2005, Ningxia began to break the industrial boundaries by an easy access to large-scale scientific equipment. It was turned into reality that the management of large-scale scientific equipment resources had shifted from the mode of "segregation of departments" to "co-construction and - sharing". Besides, Ningxia began to train managerial staff at all scientific research institutes and universities in batches, thus increasing the utilization rate to $79 \%$ and the sharing rate to $66.5 \%$. Through integration, by 2010 , the instruments included into the network of the whole region had reached 525, with 62 units involved in the network. The accumulated visits of the websites of large-scale scientific equipment amounted to 130,000 . This further highlights the importance of management mode to the increase of instruments' utilization rate and sharing rate.

\section{POLICY SUGGESTIONS}

On the basis of the survey on the utilization and sharing of large-scale scientific equipment and the analysis on the results of textual data processing, the following reflections are made:

(1) Make adjustment on the policies for the sharing of largescale scientific equipment based on differentiation.

China is a vast country with uneven development of economy, science and education in different regions. Therefore, the development level of large-scale scientific equipment is affected, and the regional differences are obvious. Corresponding adjustment on policies should be made according to the differences. For regions where the equipped level is high but the sharing rate is common, we should target the efforts to establishing and improving the system of purchasing, checking for redundancy and evaluation of large-scale scientific equipment to avoid repetitive purchase. For regions where the equipped level is low, we should make proper arrangement and adjustment in conformity with the specific supply capacity, operating state and sharing construction, strengthen the supports from the governments in the form of awards and subsidies. The negative factors should be eliminated and the capacity of sharing regional scientific resources should be enhanced.

\section{(2) Capacity building for the management professionals}

The key to ensure the normal and efficient operation of large-scale equipment and to collect high-quality basic data 
of the utilization status is the high qualification of management professionals. We should attach importance to the training of researchers, promote their experimental skills and the ability to maintain the equipment. A scientific and reasonable qualification test can promote the management department to gain a full understanding of large-scale equipment and thus further motivating the enthusiasm of the researchers. Besides, large-scale equipment requires the maintenance by professional and trained talents. So universities and enterprises should introduce talents of this kind, which is not only conducive to the efficient use of large-scale equipment, but also plays a positive role in the innovation on the basis of a good mastery of equipment operational skills. Regular lectures and technological trainings can help management staff members to improve their professional skills and technical capacity. Thus, they can do well in maintenance, reduce failure rate, and increase the time of normal running of equipment.

(3) Promote the construction of the platform of large-scale scientific equipment sharing and the establishment of open-end funds.

On one hand, we should further construct the technology platform and monitor the degree of utilization and sharing, which plays a significant role in providing references for the technology management and policy-making process. As for the existing large-scale scientific equipment which have a low utilization rate, we should constantly push forward the opening and sharing of equipment, thus generating more service income while facilitating the opening and sharing of the equipment. On the other hand, we should promote the establishment of open-end funds, such as equipment maintenance fund and testing fund which encourage researchers to use large-scale equipment and create better working conditions for the implementation of research projects. Moreover, these measures can provide enough money for the maintenance of large-scale equipment. The sharing of large-scale scientific equipment gives impetus for the increase of their utilization rate and application level.

\section{CONCLUSION}

The utilization and sharing of large-scale scientific equipment involve multiple parties including investors, users, owners and service providers, as well as relevant mechanisms and legal construction. A large amount of capital input and talent training are necessary. Therefore, the utilization and sharing of large-scale scientific equipment is a huge and complicated systematic project worthy of multidimensional analysis and assessment. The multi-dimensional model built in this article can allow different regions to learn about the problems existing in the sharing of large-scale scientific instruments. This will provide a sound basis for the regional technological resources' management and utilization and also for policy-making. Of course, the analysis in this article is quite elementary and the policies proposed are tentative. The correlation between the utilization and sharing large-scale scientific equipment, the influence factors and what a high sharing index indicates are the topics of further studies.

\section{CONFLICT OF INTEREST}

The authors declare that they have no conflict of interest.

\section{ACKNOWLEDGEMENTS}

We would like to thank Beijing Jiaotong University and Ministry of Science and Technology and Ministry of Education of the People's Republic of China. This study was sponsored by Special fund for basic platform for "Evaluation of index of utilization and sharing of large-scale scientific instruments", the program of Ministry of Science and Technology (2013DDJ1ZY06), and special fund for scientific evaluation and statistics of "Evaluation of index of utilization and sharing of large-scale scientific instruments", the program of Ministry of Science and Technology (2013SE-0603). We also would like to thank other teachers and students who provide us the data and information.

\section{REFERENCES}

[1] Ministry of Education of People's Republic of China. "National Guideline on Medium - and Long-Term Program for Science and Technology Development," (2006 - 2020)", 2006.

[2] Ministry of Science and Technology of People's Republic of China. "2004-2010 National Guideline on Infrastructure Platform Construction of Science and Technology", 2004.

[3] Y. Liu, "Discussion about the reasonable allocation and resource sharing of large-scale scientific equipment at local universities", Lab. Res. Explor., Vol. 2, pp.72-74, April 2001.

[4] C. Xia, Y. Yuan and W. Wang, "A proposition on the sharing system of large scientific instruments", Sci. Technol. Manag. Res., Vol. 3, pp. 18-24, March 2005.

[5] J. Xu and D. Wang, "Analysis on the utilization status of China's large-scale scientific equipment and policy implications", J. Eng. Stud., Vol. 3, pp. 209-216, Sep. 2010.

[6] X. Zhang, C. Han and T. Zhao, "Evaluation study on the use of large-scale scientific equipment based on rank sum model", Sci. Res. Manag., Vol. 4, pp. 108-114, 2013.

[7] H. Wang and J. Wang, "Large-scale scientific equipment in beijing and the preliminary assessment on their sharing", $C h$. Inform. Rev., Vol. 1, pp.104-109, 2014.

[8] P. Jie and T. Jie, "Statistical analysis and suggestions on utilization rate of large-scale scientific equipment", Mod. Instrum., Vol. 2, pp. 55-58, 2007.

[9] F. Ao, "Studies on and realization of virtualized remote sharing mechanism of large-scale scientific equipment", Taiyuan Univ. Technol., pp. 95, 2008. 